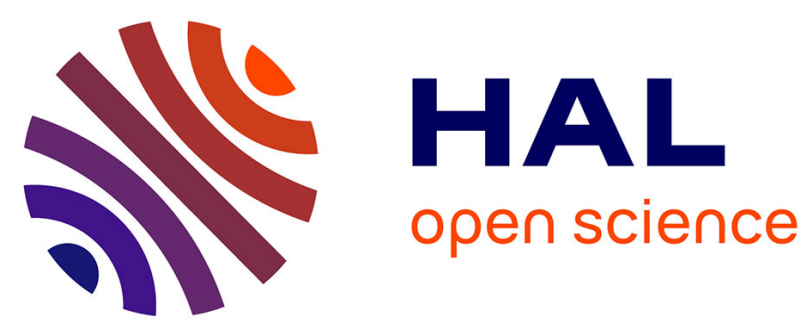

\title{
Characterization of Diblock Copolymers by Capillary Electrophoresis: From Electrophoretic Mobility Distribution to Distribution of Composition
}

\author{
Anthony Phimphachanh, Joseph Chamieh, Laurent Leclercq, Simon
}

Harrisson, Mathias Destarac, Patrick Lacroix-Desmazes, Corine Gerardin, Martin In, Hervé Cottet

\section{To cite this version:}

Anthony Phimphachanh, Joseph Chamieh, Laurent Leclercq, Simon Harrisson, Mathias Destarac, et al.. Characterization of Diblock Copolymers by Capillary Electrophoresis: From Electrophoretic Mobility Distribution to Distribution of Composition. Macromolecules, 2020, 53 (1), pp.334-345. 10.1021/acs.macromol.9b01978 . hal-02462075v2

\section{HAL Id: hal-02462075 \\ https://hal.umontpellier.fr/hal-02462075v2}

Submitted on 9 Dec 2020

HAL is a multi-disciplinary open access archive for the deposit and dissemination of scientific research documents, whether they are published or not. The documents may come from teaching and research institutions in France or abroad, or from public or private research centers.
L'archive ouverte pluridisciplinaire HAL, est destinée au dépôt et à la diffusion de documents scientifiques de niveau recherche, publiés ou non, émanant des établissements d'enseignement et de recherche français ou étrangers, des laboratoires publics ou privés. 
1 Characterization of diblock copolymers by capillary electrophoresis: From electrophoretic mobility distribution to distribution of composition

Anthony Phimphachanh ${ }^{1,3}$, Joseph Chamieh², Laurent Leclercq², Simon Harrisson ${ }^{4}$, Mathias Destarac ${ }^{4}$, Patrick Lacroix-Desmazes ${ }^{3}$, Corine Gérardin ${ }^{3}$, Martin In $^{1 *}$, Hervé Cottet ${ }^{2 *}$

${ }^{1}$ L2C, Univ Montpellier, CNRS, Montpellier, France

${ }^{2}$ IBMM, Univ Montpellier, CNRS, ENSCM, Montpellier, France

${ }^{3}$ ICGM, Univ Montpellier, CNRS, ENSCM, Montpellier, France

${ }^{4}$ IMRCP, University of Toulouse, CNRS UMR5623, Toulouse, France

*Corresponding authors: herve.cottet@umontpellier.fr \& martin.in@ umontpellier.fr

\section{Abstract}

Free solution capillary-electrophoresis (CE) is a powerful separation technique for the characterization of diblock copolymers. In this work, four series of double-hydrophilic anionic and cationic block copolymers, namely, poly(acrylamide)-block-poly(acrylic acid) (PAM-b-PAA), poly(acrylamide)-block- poly((3-acrylamidopropyl)trimethylammonium chloride) (PAM- $b$-PAPTAC), poly(ethylene oxide)-block-poly(acrylic acid) (PEO-b-PAA) and poly(poly(ethylene glycol) methyl ether acrylate)-block-poly(acrylic acid) (P(PEGA)-b-PAA), were synthesized by reversible additionfragmentation chain transfer (RAFT) polymerization and characterized by CE. The electrophoretic mobility distributions of the copolymers were transformed into distributions of composition ratio by introducing a retardation parameter, $X_{\text {exp, }}$, that represents the hydrodynamic drag retardation due to the neutral block of the copolymer. A linear correlation between $X_{\exp }$ and the ratio of the degrees of polymerization of each blocks was experimentally established and was consistent with the model of electrophoretic mobility of composite macromolecules with hydrodynamic coupling. Finally, the comparison of the distributions between the different copolymer families was significantly improved by considering the distributions in composition ratio compared to the electrophoretic mobility distributions, since it takes into account the differences in solvation, expansion and drag force according to the chemical nature of the blocks. 
The characterization of diblock copolymers by separation techniques is challenging but of primary importance to check their purity and to characterize their distributions in size and in chemical composition. ${ }^{1}$ Block copolymers prepared by reversible deactivation radical polymerization frequently contain homopolymer impurities. ${ }^{2,3}$ These include dead chains from termination reactions during polymerization of the first block, as well as the products of side-reactions such as chain transfer to solvent or monomer during polymerization of the second block. The growth of the second block in copolymer synthesis is often evidenced by a decrease of the elution time in size-exclusion chromatography (SEC) as a result of the increased hydrodynamic radius ${ }^{4,5}$. However, this only holds if the second block contributes significantly to the hydrodynamic radius of the diblock copolymer. Getting more quantitative information can be challenging especially in aqueous SEC. Different solvation properties between the blocks of a block copolymer can lead to coelution of polymers of different mass in SEC, resulting in inaccuracy in the obtained molar masses. ${ }^{6}$ Additionally, interactions with the stationary phase $\mathrm{s}^{3}$ may lead to HPLC-type elution which is dependent on the chemical composition of the polymer as well as its size. For diblock copolymer SEC, particular elution conditions are generally required and size distributions should be expressed in terms of hydrodynamic radius (and not molar mass) due to the difference in chemical composition / solvation of the two blocks ${ }^{6}$. The proportion of each monomer in a copolymer can be obtained by liquid chromatography under critical conditions LCCC, also known as LC-PEAT, for the point of exclusion-adsorption transition for neutral blocks ${ }^{7,8}$. The critical conditions for LCCC (or LC-PEAT) are usually difficult to find and are very sensitive to small changes in mobile phase composition and/or temperature. An alternative separation technique for charged copolymers is free solution capillary electrophoresis (CE) ${ }^{2,3,7-9}$. The electrophoretic separation of charged homopolymers from diblock copolymers is generally easily obtained in free solution CE. Moreover, for self-assembling diblock copolymers, CE can also separate micelles from unimers ${ }^{2,7,8}$ and allows studying the impact of added surfactant on the copolymer micelles ${ }^{2,7}$. In the presence of cationic blocks, experimental difficulties 
arise from polymer adsorption onto the wall of silica based capillaries. The characterization of cationic diblock copolymers requires the use of a neutrally coated ${ }^{3}$, or positively charged capillary ${ }^{10}$. Another challenging issue in the characterization of diblock copolymers by $\mathrm{CE}$ is to extract the distribution in composition of the copolymers from the electropherogram. Raw electropherograms can be transformed into distributions of effective mobility, or of any other related parameter, provided that the relationship between effective mobility and the considered parameter is known ${ }^{11}$. The determination of polymer dispersity via the variance of the chemical composition distribution ${ }^{11}$ or via the calculation of the ratio of moments of the distribution has also been studied ${ }^{1}$. A key point to achieve such electropherogram transformation is to have a reliable relation between the effective mobility of the diblock copolymer and the degrees of polymerization of each block and thus, to the chemical composition of the copolymer ${ }^{3}$. The electrophoretic mobility of a diblock copolymer is generally expressed as a weighted average of the mobilities of different subunits constituting the copolymer ${ }^{12}$. The choice of the subunits and the corresponding weights have been described in the literature as depending on the conformations of each block and on the hydrodynamic coupling regime between the two blocks 12-15. More recently, Chubynsky and Slater studied in more detail the "end-effect" (i.e. the fact that the ends of the copolymer chain are more hydrodynamically exposed to the solvent $)^{16}$ and the effect of polymer stiffness on the electrophoretic modeling ${ }^{17}$. The electrophoretic models of composite objects ${ }^{12,}$ 13 which are relevant for diblock copolymers, were also applied to end-labeled free solution electrophoresis (ELFSE) ${ }^{14,15}$, which consists in attaching a monodisperse neutral block (drag-tag) to a polydisperse biopolyelectrolyte (for instance, for DNA sequencing in free solution ${ }^{15}, 18$ ), or conversely, in attaching a monodisperse polyelectrolyte to a polydisperse neutral polymer (for instance, for sizebased neutral polymer characterization ${ }^{14}$ ). In this way, the dependence of electrophoretic mobility with the molar mass of the end-labelled composite object is obtained in free solution due to the variation of the charge-to-friction ratio.

Double-hydrophilic block copolymers (DHBC) are block copolymers containing two hydrophilic segments. DHBCs on their own are completely soluble in water and do not self-assemble in dilute conditions. DHBCs can still retain an amphiphilic character and this can lead to self-organization at the meso-scale in concentrated conditions ${ }^{19}$. They can undergo morphological transitions induced by 
external stimuli ${ }^{20}$ in dilute solution. The great development of reversible deactivation radical polymerization $^{21-24}$ in the last two decades allows tailoring the stimuli-responsiveness (e.g. to changes in $\mathrm{pH}$, temperature, ionic strength, or light) of these polymers by controlling both the nature of monomers and the degree of polymerization of the blocks. When one block is a polyelectrolyte, DHBCs can undergo micellization by electrostatic complexation in the presence of an oppositely charged polyelectrolyte. These properties lead to a wide range of applications such as control of crystallization of inorganic compounds ${ }^{25}$, drug delivery ${ }^{26}$ or template for ordered mesoporous materials ${ }^{27}$. For this last application of DHBC, the asymmetry ratio, defined as the ratio of degrees of polymerization of both blocks, is of crucial interest since it determines the structure of the DHBC-templated mesoporous materials.

It is the aim of the present work to characterize the composition of DHBC by CE, with particular attention to the asymmetry ratio. The approach has been applied to series of anionic and cationic DHBCs, namely, poly(acrylamide)-block-poly(acrylic acid) (PAM-b-PAA), poly(acrylamide)-blockpoly((3-acrylamidopropyl)trimethylammonium chloride) (PAM-b-PAPTAC), poly(ethylene oxide)block-poly(acrylic acid) (PEO-b-PAA) and poly(poly(ethylene glycol) methyl ether acrylate)-blockpoly(acrylic acid) (P(PEGA)-b-PAA). These DHBC have been synthesized in aqueous medium by reversible addition-fragmentation transfer (RAFT) polymerization.

In the next section, different models for the electrophoretic mobility of composite objects such as block copolymers are briefly reviewed. In the third section, the synthesis of the copolymers and the experimental conditions of their characterization by $\mathrm{CE}$ are reported. The results of this work are presented in section 4, where we describe a method to transform the distribution of electrophoretic mobility into a distribution of the ratio of degree of polymerization of both blocks. 
- Neutral part $\quad$ Polyelectrolyte part

Model 1

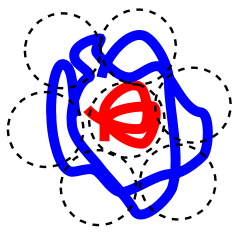

Hydrodynamic coupling
Model 2

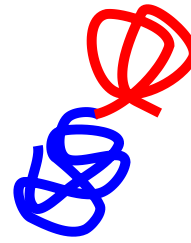

Segregation/coil
Model 3

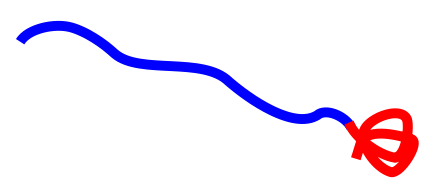

Segregation/stretch

Figure 1. Representation of possible conformations of a double hydrophilic block copolymer composed of a neutral Gaussian coil (in red) linked to a polyelectrolyte block (in blue). In model 1, the polyelectrolyte chain is a coil in hydrodynamic interaction with the neutral Gaussian coil. In model 2, the polyelectrolyte and the neutral polymer separate into two coils. In model 3, the polyelectrolyte is fully stretched and has no hydrodynamic coupling with the neutral coil. Adapted from ${ }^{28}$

In this section, we present different possible electrophoretic mobility models that are relevant for diblock copolymers composed of a polyelectrolyte part attached to a neutral polymer coil. These theoretical models were developed by Desruisseaux et $\mathrm{al}^{28}$, building on previous work by Long et al. ${ }^{12 \text {, }}$

${ }^{13}$ Figure 1 shows the different possible conformations that can be encountered for DHBC, corresponding to the different models presented below.

\subsection{Model 1: hydrodynamic coupling}

In Model 1, hydrodynamic coupling between the polyelectrolyte part and the neutral coil is taken

111 into account. The polyelectrolyte block of the DHBC is composed of $N_{\text {blob }}$ equivalent blobs of a size equivalent to the hydrodynamic radius of the neutral coil $R_{h}^{\text {neutral }}$. If $R_{h}^{\text {neutral }}$ is larger or equal to the

113 Debye length, Long et al. ${ }^{29}$ demonstrated that the electrophoretic mobility of the DHBC composite object composed of $N_{b l o b}+1$ subunits of equal size, is given by the number-average of the electrophoretic mobilities calculated on all the equivalent blobs constituting the object. The electrophoretic mobility of the DHBC, $\mu_{e p, 1}^{\text {diblock }}$, is thus given by ${ }^{28}$ : 
$\mu_{e p, 1}^{\text {diblock }}=\frac{\sum_{i=0}^{N_{\text {blob }}} \mu_{e p}^{i}}{N_{\text {blob }}+1}=\frac{N_{b l o b} \mu_{e p}^{0}+\mu_{e p}^{\text {neutral }}}{N_{\text {blob }}+1}=\frac{\mu_{e p}^{0}}{1+\frac{\alpha}{D P_{0}}}$

118 where $\mu_{e p}^{i}$ is the effective mobility of the $i^{\text {th }}$ entity (or blob) constituting the DHBC, $D P_{0}$ is the degree

119 of polymerization of the polyelectrolyte block, $\alpha$ is the number of charged monomers per blob, $\mu_{e p}^{0}$ is

120 the effective electrophoretic mobility of the polyelectrolyte part (alone) and $\mu_{e p}^{\text {neutral }}$ is the electrophoretic

121 mobility of the neutral part $\left(\mu_{e p}^{\text {neutral }}=0\right)$. The number of equivalent blobs in the polyelectrolyte chain

122 is given by $N_{b l o b}=\frac{D P_{0}}{\alpha}$. Note that $N_{b l o b}$ (and $\mu_{e p, 1}^{\text {diblock }}$ ) depends on the polyelectrolyte persistence

123 length, and thus, on the ionic strength. Equation (1) neglects the so-called end-effect ${ }^{16}$. Please note that

124 subscript and superscript 0 refer to the polyelectrolyte block, for consistency with ref 28.

\subsection{Models without hydrodynamic coupling}

\subsubsection{Model 2: polyelectrolyte chain in coil conformation}

Model 2 in Figure 1 corresponds to the segregation of the neutral polymer coil from the polyelectrolyte coil. In the absence of hydrodynamic coupling between the two parts, and if the polyelectrolyte chain does not stretch during electrophoresis (i.e. at sufficiently low electric field), the electrophoretic mobility of the DHBC, $\mu_{e p, 2}^{\text {diblock }}$, is given by the average electrophoretic mobility of the two parts weighted by their hydrodynamic friction coefficient ${ }^{12}$. Using Stokes equation for spherical objects, $\mu_{e p, 2}^{\text {diblock }}$ is expressed as ${ }^{13,28}$ :

$\mu_{e p, 2}^{\text {diblock }}=\frac{\sum_{i=0}^{1} \gamma_{i} \mu_{e p}^{i}}{\sum_{i=0}^{1} \gamma_{i}}=\frac{\mu_{e p}^{0}}{1+\frac{R_{h}^{\text {enutral }}}{R_{h}^{0}}}$

134 where $\gamma_{i}$ is the friction coefficient of the $i^{\text {th }}$ part constituting the DHBC, $R_{h}^{\text {neutral }}$ is the hydrodynamic

135 radius of the neutral coil, $R_{h}^{0}$ is the hydrodynamic radius of the polyelectrolyte block. 


\subsubsection{Model 3: polyelectrolyte chain in fully stretched conformation}

$$
\mu_{e p, 3}^{\text {diblock }}=\frac{\sum_{i=0}^{1} \gamma_{i} \mu_{e p}^{i}}{\sum_{i=0}^{1} \gamma_{i}}=\frac{\mu_{e p}^{0}}{1+\frac{2 R_{h}^{\text {neutral }}}{b_{0} D P_{0}} \ln D P_{0}}
$$

where $b_{0}$ is the size of a charged monomer in the polyelectrolyte chain. Equation (3) assumes that the

144 friction coefficient $\gamma_{\text {rod }}$ of the stretched polyelectrolyte cylinder is averaged on all orientations relative to the flow direction, and is given by:

146

$$
\gamma_{\text {rod }}=\frac{3 \pi \eta b_{0} D P_{0}}{\ln \left(D P_{0}\right)}
$$

147 where $\eta$ is the viscosity of the solvent.

148 Even if the electrical field is not strong enough to stretch the polyelectrolyte block (hydrodynamic 149 segregation), the polyelectrolyte contour length may still be shorter than the persistence length of the polyelectrolyte. In this situation, the segregation between the neutral and the polyelectrolyte parts is sterically obtained, but the electrophoretic mobility is still described by equation $(3)^{27}$.

\section{Experimental}

\subsection{Chemicals}

Ammonium persulfate (APS, 98\%) and sodium formaldehyde sulfoxylate dehydrate (NaFS, 98\%) were purchased from Acros organics. Poly(ethylene glycol) methyl ether acrylate $\left(\mathrm{M}_{\mathrm{n}}=480 \mathrm{~g} / \mathrm{mol}, 8.5 \mathrm{EO}\right.$ units on average) and (3-acrylamidoprpyl)trimethylammonium chloride (APTAC) aqueous solution (75 
wt.\%) were purchased from Sigma Aldrich (Saint-Quentin-Fallavier, France) and used as received. Acrylic acid from the same suppliers was distilled under vacuum at room temperature. PEO $M_{n}=5000$ g. $\mathrm{mol}^{-1}, \mathrm{D}=1.04$ was purchased from. 4,4'-azobiscyanopentanoic acid (ACPA, Aldrich, 98\%) and 2,2Azobis(isobutyramidine) dihydrochloride (AIBA, Sigma-Aldrich, 97\%) were used as received.

For EC experiments, background electrolytes were prepared in ultra-pure water purified on a Millipore system (Molsheim, France) from tris(hydroxymethyl)aminomethane (TRIS, 99,9\%, Merck), 4Morpholinoethanesulphonic acid (MES, >99\%, Acros Organics), and 2-[Bis(2-hydroxyethyl)amino]-2(hydroxymethyl)propane-1,3-diol (BIS-TRIS, >99\%, Acros Organics). Anisic acid (99,5\%), ammediol $(99,5 \%)$ used as markers for detection were purchased from Sigma-Aldrich.

\subsection{Double-hydrophilic block copolymer synthesis}

This section describes the synthesis of the copolymers. The synthetic pathway, the size exclusion chromatograms and the ${ }^{1} \mathrm{H}$ NMR spectra are given in SI as well as the temporal electropherograms.

\subsubsection{Synthesis of poly(acrylamide)-b-poly(acrylic acid)}

\section{Aqueous RAFT/MADIX polymerization of AA}

Polyacrylamide macro RAFT/MADIX agent (PAM-Xa, $\mathrm{M}_{\mathrm{n}}=5000 \mathrm{~g} \cdot \mathrm{mol}^{-1}$ ) was synthesized according the procedure described by Layrac et al.. ${ }^{30}$ Synthesis of $\mathrm{PAM}_{70}-b-\mathrm{PAA}_{20}$ was performed as follows ${ }^{31}$ : PAM-based chain transfer agent (PAM-Xa,) (15.76 g, 3.029 mmol), acrylic acid (4.24 g; 58.77 mmol), AIBA $(0.0821 \mathrm{~g}, 0,3 \mathrm{mmol})$ and water $(42 \mathrm{~g})($ solids $=30.6 \%)$ were introduced in a round bottom flask. The mixture was degassed with argon at room temperature for $30 \mathrm{~min}$ and then placed in a thermostated oil bath at $65^{\circ} \mathrm{C}$ under argon for 2 hours. Conversion was quantitative, acrylic acid traces were eliminated by dialysis (MWCO $1000 \mathrm{Da}$ ) and pH-metric monitoring. The polymer solution was then freeze dried and a white powder was obtained. Four DHBC were synthesized according to this procedure: $\mathrm{PAM}_{70}-b-\mathrm{PAA}_{20}, \mathrm{PAM}_{140}-b-\mathrm{PAA}_{40}, \mathrm{PAM}_{140}-b$ - $\mathrm{PAA}_{60}$ and $\mathrm{PAM}_{140}-b-\mathrm{PAA}_{80}$ (see Table 1). 


\subsubsection{Synthesis of poly(acrylamide)-b-poly((3-acrylamidopropyl)trimethylammonium chloride)}

\section{Aqueous redox RAFT/MADIX polymerization of APTAC}

Synthesis of $\mathrm{PAM}_{70}-b$-PAPTAC 30 was performed as follows: two aqueous solutions of NaFS (5\%w) (1.23 g of solution, $0.4 \mathrm{mmol})$ and $\mathrm{NaPS}(5 \% \mathrm{w})(2.37 \mathrm{mg}$ of solution, $0.5 \mathrm{mmol})$ were prepared. PAMXa macroxanthate $(9.97 \mathrm{~g}, 1.99 \mathrm{mmol})$, APTAC monomer (13.3 $\mathrm{g}$ of solution, $0.057 \mathrm{mmol})$, and water $(50 \mathrm{~g})($ solids $=25.4 \%)$ were introduced in a round bottom flask. The $\mathrm{pH}$ of the mixture was first adjusted at 2 with hydrochloric solution $1 \mathrm{M}$. Then the mixture was degassed with argon at room temperature for 30 min and placed in a thermostated oil bath at $25^{\circ} \mathrm{C}$ under argon. Both solution of NaFS and NaPS were introduced in the round bottom flask and the reaction mixture was stirred for 3 hours. Monomer traces were eliminated with dialysis (MWCO $1000 \mathrm{Da}$ ) and conductivity monitoring. The polymer solution was then lyophilized and a white powder was obtained. This redox process at $25^{\circ} \mathrm{C}$ was developed after the paper of Sutton et al. ${ }^{9}$ to minimize the formation of dead chains. Four DHBC were synthesized according to this procedure: $\mathrm{PAM}_{70}-b-\mathrm{PAPTAC}_{30}, \mathrm{PAM}_{70^{-}} b-\mathrm{PAPTAC}_{60}, \mathrm{PAM}_{140^{-}} b-$ PAPTAC $_{60}$ and PAM $_{140}-b-$ PAPTAC $_{120}$ (see Table 1).

\subsubsection{Synthesis of poly(ethylene oxide)-b-poly(acrylic acid)}

\section{Aqueous RAFT polymerization of AA}

Synthesis of $\mathrm{PEO}_{105}-b-\mathrm{PAA}_{20}$ was performed as follows: $\mathrm{PEO}_{105}-\mathrm{CTA}$ (poly(ethylene oxide)-chain transfer agent) macro RAFT agent was obtained following the procedure published by Bathfield et $\mathrm{al}^{32}$. PEO ${ }_{105}-\mathrm{CTA}$, (10.53 g, $\left.2.19 \mathrm{mmol}\right)$, ACPA (0.123 mg, 0,439 mmol), acrylic acid (5.5 g, $\left.76 \mathrm{mmol}\right)$ and deionized water $(29.5 \mathrm{~mL})$ (solids=35.4\%) were introduced in a Schlenk tube equipped with a magnetic stirrer. The mixture was degassed by five freeze-evacuate-thaw cycles and then heated for 42 hours at $75^{\circ} \mathrm{C}$ under nitrogen in a thermostated oil bath. Final conversion $=72 \%$. Monomer conversion was determined by ${ }^{1} \mathrm{H}$ NMR spectroscopy, using a Bruker 400MHZ spectrometer. Samples for analysis by NMR were prepared by adding $0.6 \mathrm{~mL}$ of $\mathrm{D}_{2} \mathrm{O}$ to $0.1 \mathrm{~mL}$ of polymerization medium. Once the reaction was complete, the solvent was evaporated, and then the DHBC was dissolved in a minimum amount of dichloromethane before being precipitated twice in a large volume of cold diethyl ether. It was then recovered by filtration, and finally dried under vacuum overnight before analysis by SEC and ${ }^{1} \mathrm{H}-\mathrm{NMR}$. SEC was performed in DMF-LiBr after methylation ${ }^{33}$ with trimethylsilyldiazomethane. Four DHBC 
were synthesized according to this procedure: $\mathrm{PEO}_{105}-b-\mathrm{PAA}_{20}, \mathrm{PEO}_{105}-b-\mathrm{PAA}_{30}, \mathrm{PEO}_{210}-b-\mathrm{PAA}_{40}$ and

$\mathrm{PEO}_{210}-b-\mathrm{PAA}_{50}$ (see Table 1). The degrees of polymerization of the commercial starting PEO have

212 been determined by ${ }^{1} \mathrm{H} \mathrm{NMR}^{32}$ and are presented in Table 1.

\subsubsection{Synthesis of poly(acrylic acid)-b-poly(poly(ethylene glycol) methyl ether acrylate)}

\section{Aqueous RAFT polymerization of AA and PEGA}

5,7-dithia-6-thio-4-methyl-4-cyanodecanoic acid (CTPPA) was obtained by reaction of ACPA with bis(propylsulfanylthiocarbonyl) disulfide according to literature. ${ }^{34}$ Synthesis of $\mathrm{PAA}_{21}-b$ P(PEGA $)_{12}$ was performed as follows: PAA-CTPPA chain transfer agent was synthesized according to the process described in literature ${ }^{35}$ : in a round bottom flask, CTPPA $(0.8 \mathrm{~g}, 2.57 \mathrm{mmol}$, purity=89\%), acrylic acid $(3.9 \mathrm{~g}, 54.1 \mathrm{mmol})$, ACPA $(0,072 \mathrm{~g}, 0.26 \mathrm{mmol})$ and half of the amount of water $(7.5 \mathrm{~g})$ are stirred until dissolution of CTPPA. The remaining water (7.5 g) was introduced and the mixture was degassed with argon for $40 \mathrm{~min}$. The mixture was then heated in an oil bath at $70^{\circ} \mathrm{C}$ for $5.5 \mathrm{~h}$. For the synthesis of PAA- $b$-P(PEGA), ACPA $(0.0715 \mathrm{~g} ; 0.26 \mathrm{mmol})$ and poly(ethylene glycol) methyl ether acrylate $(12.8 \mathrm{~g}, 26.4 \mathrm{mmol})$ were added to the PAA-CTPPA reaction medium and the mixture was degassed with argon for $40 \mathrm{~min}$. The mixture was then heated in an oil bath at $70^{\circ} \mathrm{C}$ for $5 \mathrm{~h}$. Conversion was followed by ${ }^{1} \mathrm{H}$ NMR. At the end of the polymerization, water was evaporated under reduced pressure and the polymer washed with diethyl ether. NMR sample preparation: $0.6 \mathrm{~mL}$ of $\mathrm{D}_{2} \mathrm{O}$ was added to $0.1 \mathrm{~mL}$ of polymerization medium and quenched in liquid nitrogen. Two DHBC were synthesized according to this procedure: $\mathrm{P}(\mathrm{PEGA})_{12}-b-\mathrm{PAA}_{21}$ and $\mathrm{P}(\mathrm{PEGA})_{22}-b-\mathrm{PAA}_{45}$ (see Table 1$)$.

Table 1: Presentation of the chemical structure and the different DHBC samples synthesized and studied in this work. The subscripts in the names correspond to the degree of polymerization of each block. $M W_{\text {neutral }}$ and $M W_{0}$ are the molar masses of the neutral and of the polyelectrolyte block respectively.

\begin{tabular}{ccc}
\hline Type of DHBC & $\begin{array}{c}M W_{\text {neutral }}-M W_{0} \\
\text { as determined by } \\
\text { NMR }\end{array}$ & Chemical structure \\
& $5 \mathrm{k}-1.4 \mathrm{k}$ \\
\hline $\mathrm{PAM}_{70}-b-\mathrm{PAA}_{20}$ & $10 \mathrm{k}-2.8 \mathrm{k}$
\end{tabular}


$\mathrm{PAM}_{140} b-\mathrm{PAA}_{60}$

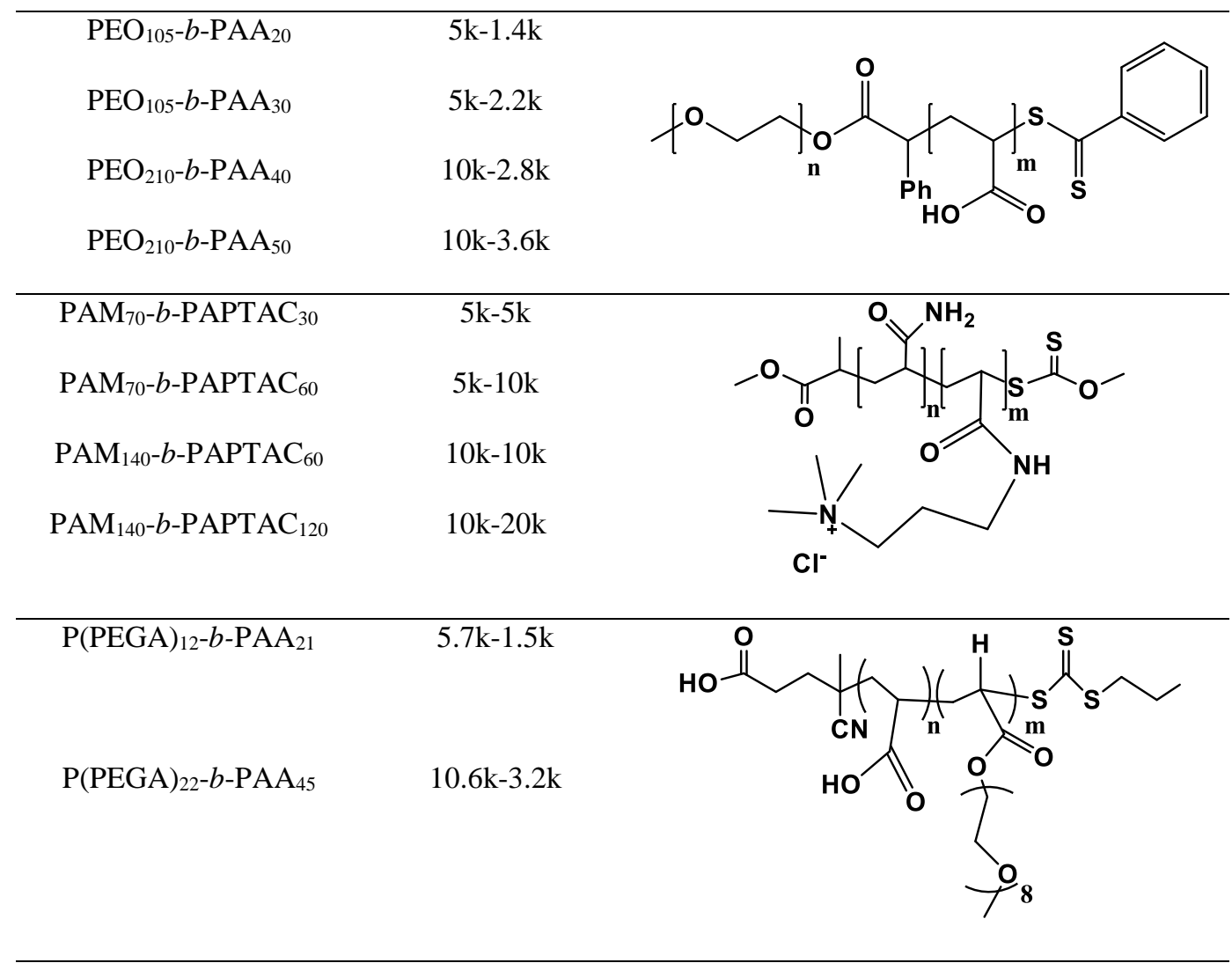

\subsection{Capillary electrophoresis}

\section{Instrumentation and method}

234 Capillary electrophoresis experiments were performed on an Agilent 7100 capillary electrophoresis 235 instrument with a diode array UV detector. Fused silica capillaries of 50/375 $\mu$ m I.D./O.D. with 236 polyimide outer coating (cat. no. TSP050375) were from Polymicro Technologies (Phoenix, AZ, USA).

237 Capillary dimensions were $38.5 \mathrm{~cm}$ long ( $30 \mathrm{~cm}$ to detection window). New capillaries were conditioned 238 by performing the following washes at 1 bar: $1 \mathrm{M} \mathrm{NaOH}$ for $30 \mathrm{~min}$ and water for $15 \mathrm{~min}$. The temperature of the capillary cartridge was set at $25^{\circ} \mathrm{C}$.

In the case of PAM- $b$-PAA, an electrolyte consisting of $20 \mathrm{mM}$ MES and $14 \mathrm{mM}$ ammediol $\mathrm{pH} 6.5$ was used. $0.1 \mathrm{~g} / \mathrm{L}$ anisic acid was added in the sample as a mobility marker. The same background electrolyte

242 was used for PAM- $b$-PAPTAC, but with a different mobility marker (imidazole $0.1 \mathrm{~g} / \mathrm{L}$ ). In the case of PEO- $b$-PAA and P(PEGA)- $b$-PAA, an electrolyte constituted of $6 \mathrm{mM}$ anisic acid and $12 \mathrm{mM}$ BIS- 
244 TRIS, pH 6.5 was used as buffer, with MES at $0.5 \mathrm{~g} / \mathrm{L}$ as mobility marker in the case of P(PEGA)-b245 PAA.

246 All copolymers were dissolved in water at a concentration of $5 \mathrm{~g} / \mathrm{L}$. Samples were injected 247 hydrodynamically on the inlet side of the capillary by applying $30 \mathrm{mbar}$ for $5 \mathrm{~s}$. Separations were carried 248 out by applying $a+20 \mathrm{kV}$ voltage. For PAM- $b$-PAA, PEO- $b$-PAA and P(PEGA)- $b$-PAA and PAM- $b$ -

249 PAPTAC, detection was realized at $192+/-2 \mathrm{~nm}$ (reference off).

250 For PAM- $b$-PAA, PEO- $b$-PAA and P(PEGA)- $b$-PAA, the capillary was rinsed between each run by 251 flushing the capillary for 2 min with the background electrolyte, 2 min with $0.1 \mathrm{M} \mathrm{NaOH}, 2$ min with 252 ultra-pure water and 2 min with background electrolyte. For the analysis of the cationic polymer PAM$253 b$-PAPTAC, and in order to reduce the adsorption on the capillary wall, surface of the capillary was 254 modified using UltraTrol ${ }^{\mathrm{TM}}$ LN (Target Discovery, Inc., Palo Alto, CA), which is a commercial neutral 255 semi-permanent coating based on polyacrylamide derivatives. The coating procedure was performed 256 using the following successive flushes: methanol for $2 \mathrm{~min}$ at $1 \mathrm{bar}$, water for $2 \mathrm{~min}$ at $3 \mathrm{bar}, 1 \mathrm{M} \mathrm{NaOH}$ 257 for $2 \mathrm{~min}$ at $3 \mathrm{bar}, 0.1 \mathrm{M} \mathrm{NaOH}$ for $2 \mathrm{~min}$ at $1 \mathrm{bar}, 1 \mathrm{M} \mathrm{HCl}$ for $5 \mathrm{~min}$ at 1 bar, water for $5 \mathrm{~min}$ at 1 bar, 258 UltraTrol ${ }^{\mathrm{TM}} \mathrm{LN}$ solution for $5 \mathrm{~min}$ at 1 bar, wait for $5 \mathrm{~min}$, water for $2 \mathrm{~min}$ at 1 bar. Prior to each analysis 259 of PAM- $b$-PAPTAC, the capillary was rinsed with the background electrolyte for 2 min at 1 bar. 


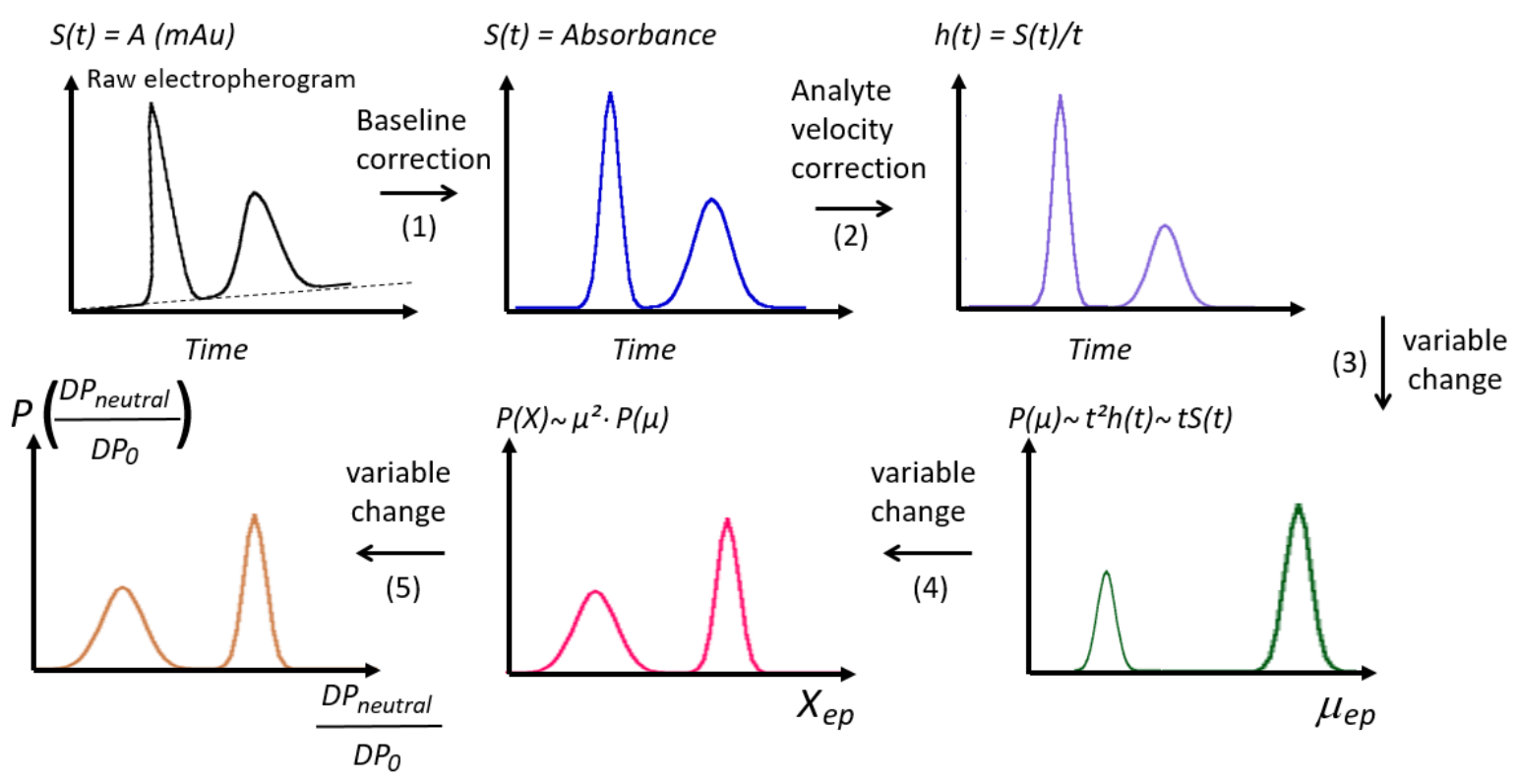

Figure 2. General scheme for changing a time-scale electropherogram into a mobility-scale and $X_{\exp }$-scale distributions. The raw electropherogram is first corrected from baseline shift (1). The time-scale electropherogram is then corrected from the differences in analyte velocities (2). The time-corrected electropherogram is converted into the effective mobility-scale distribution (3). The mobility-scale electropherogram is changed into a $X_{\text {exp }}$-scale distribution (4) and finally to a compositional $\frac{D P_{\text {neutral }}}{D P_{0}}$ ratio (5). $S(t)$ is the UV absorbance signal (in mAU). $h(t)$ is the time-corrected UV absorbance. $P\left(\mu_{e p}\right)$ is the effective mobility distribution. $P\left(X_{\exp }\right)$ is the distribution in $X_{\exp }$ (see section 4.2) and $P\left(\frac{D P_{\text {neutral }}}{D P_{0}}\right)$ is the distribution in $\frac{D P_{\text {neutral }}}{D P_{0}}$. Adapted from ${ }^{11}$ for the characterization of diblock copolymers.

Scale transformations. In this section, the transformations of the electropherograms into distributions of the parameter of interest are described following a previously published protocol ${ }^{11}$. Briefly, experimental raw time-scale electropherograms, were first corrected from any baseline shift using

265 Origin (Origin 2016, OriginLab, USA) as depicted in step 1, Figure 2. For quantitative purpose, the absorbance signal $S(t)$ was next divided by the migration time $(t)$ to correct the differences in analyte 
velocity (Figure 2, step 2$)^{11}$. Next, the time-corrected electropherogram $h(t)$ was changed into an effective mobility distribution $P\left(\mu_{e p}\right)=t \times S(t)$ (Figure 2 , step 3$)^{11}$, which requires the transformations of both the $x$ and $y$ axis $^{11}$. Note that $\mu_{e p}$ is obtained by equation (5):

$\mu_{e p}=\frac{l L}{V}\left(\frac{1}{t}-\frac{1}{t_{e o}}\right)$

271

$\overline{\mu_{e p}^{\text {diblock }}}=\frac{\int P\left(\mu_{e p}\right) \mu_{e p} d \mu_{e p}}{\int P\left(\mu_{e p}\right) d \mu_{e p}} \approx \frac{\sum_{i} P\left(\mu_{e p, i}\right) \mu_{e p, i}\left(\mu_{e p, i+1}-\mu_{e p, i}\right)}{\sum_{i} P\left(\mu_{e p, i}\right)\left(\mu_{e p, i+1}-\mu_{e p, i}\right)}$

where integration is carried out over the peak. In practice the integration is done numerically and the $i$

278 index represents the digitized experimental data points. The summation is carried out over values of $P\left(\mu_{e p, i}\right)$ greater than the median of the base line added to its standard deviation. Calculation of $\overline{\mu_{e p}^{\text {diblock }}}$ was performed using Excel 2016 (Microsoft, USA), following the discrete form of equation (6).

281 Variance of the diblock electrophoretic mobility was obtained by the following equation:

$\sigma_{\mu}{ }^{2}=\frac{\int P\left(\mu_{e p}\right)\left(\mu_{e p}-\overline{\mu_{e p}^{\text {diblock }}}\right)^{2} d \mu_{e p}}{\int P\left(\mu_{e p}\right) d \mu_{e p}}=\frac{\sum_{i} P\left(\mu_{e p, i}\right)\left(\mu_{e p, i}-\overline{\mu_{e p}^{\text {diblock }}}\right)^{2}\left(\mu_{e p, i+1}-\mu_{e p, i}\right)}{\sum_{i} P\left(\mu_{e p, i}\right)\left(\mu_{e p, i+1}-\mu_{e p, i}\right)}$ 


\section{Results and discussion}

285

286

287

288

\subsection{Electrophoretic separation of the DHBC}

The main goal of the CE characterization is to provide information about the chemical composition distribution of the DHBC and about the purity of the DHBC in terms of possible presence of homopolymers. The separation of the DHBC by CE requires an appropriate background electrolyte, depending on the nature of the copolymer. For copolymers absorbing in UV (i.e. those with a PAM neutral block), direct UV detection was possible and a background electrolyte based on $20 \mathrm{mM}$ MES and $14 \mathrm{mM}$ ammediol at pH 6.5 was used, with a UV detection at $192 \mathrm{~nm}$. For DHBC copolymers that do not absorb UV enough to ensure sensitivity (i.e. PEO- $b$-PAA or P(PEGA)- $b$-PAA), an indirect detection mode based on a $6 \mathrm{mM}$ anisic acid and $12 \mathrm{mM}$ BIS TRIS at $\mathrm{pH} 6.5$ was used. At this $\mathrm{pH}$ about $60 \%$ of the carboxylic acid groups of the PAA are ionized, and this ensures appropriate selectivity of separation between PAA homopolyelectrolyte and the DHBC. Uncoated fused silica capillary was used for the characterization of all anionic DHBC. Semi-permanent UltraTrolLN neutral coating was used for the characterization of the cationic PAM- $b$-PAPTAC DHBC, to avoid any copolymer adsorption on the capillary surface. To correct the apparent mobility from the electroosmotic mobility, a mobility marker (anisic acid for PAM- $b$-PAA, MES for P(PEGA)- $b$-PAA, and imidazolium for PAM- $b$ PAPTAC) of known effective mobility $\left(\mu_{e p, M E S}=-28 \mathrm{TU}\right.$ (where TU, Tiselius Unit, stands for $10^{-9} \mathrm{~m}^{2} \mathrm{~V}^{-}$ ${ }^{1} \mathrm{~s}^{-1}$ ) and $\mu_{e p \text {, imidazolium }}=52 \mathrm{TU}$ ) was co-injected. For POE- $b$-PAA, the electroosmotic mobility was estimated from the electroosmotic flow (EOF) peak. The distributions of effective mobility (DEM) of PAM- $b$-PAA, PEO- $b$-PAA, P(PEGA)- $b$-PAA and PAM- $b$-PAPTAC are displayed in Figure 3.
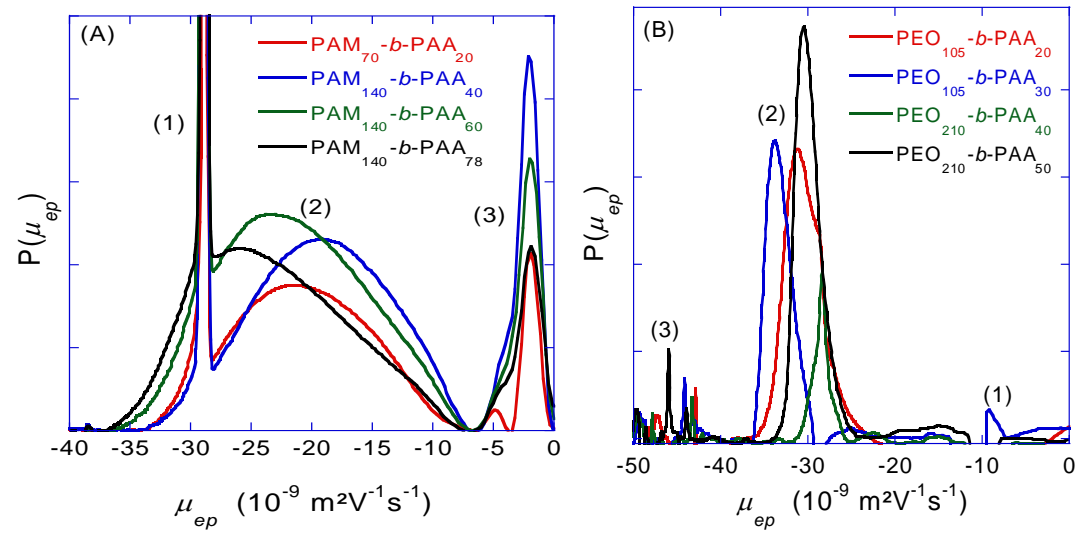

304 

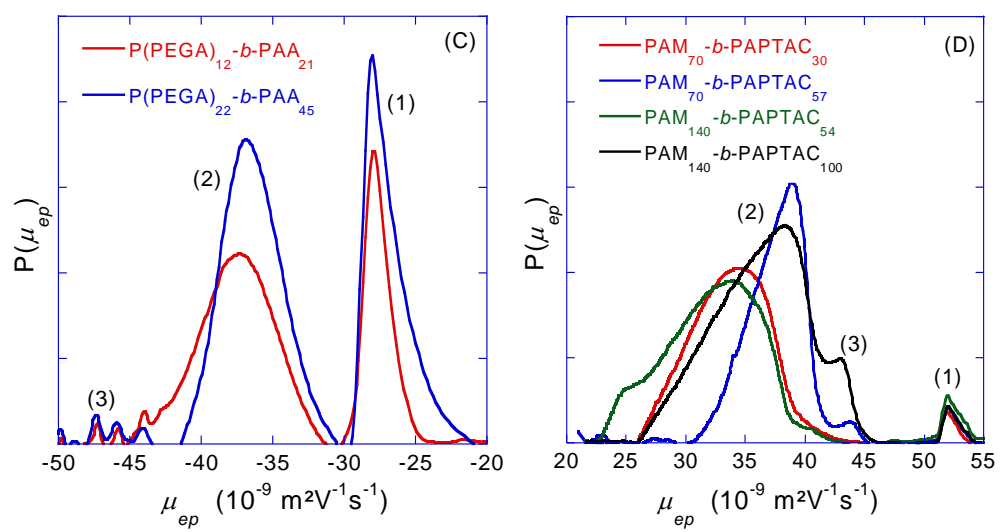

Figure 3.: Distributions of effective electrophoretic mobility obtained for PAM- $b$-PAA (A), PEO- $b$ PAA (B), P(PEGA)-b-PAA (C), and PAM- $b$-PAPTAC (D).-Electrophoretic conditions: fused silica capillary (A, B, C) or coated with UltraTrolLN ${ }^{\mathrm{TM}}$ (D), $50 \mu \mathrm{m}$ I.D. $\times 38.5 \mathrm{~cm}$ (effective length, $30 \mathrm{~cm}$ ). Electrolytes: 20 mM MES, 14 mM ammediol, pH 6.5 (A, D); 6 mM anisic acid, 12 mM BIS TRIS, pH 6.5 (B, C). Applied voltage: $+20 \mathrm{kV}$. Hydrodynamic injection: 30 mbar, 5 s. Direct (A, D) or indirect (B, C) UV detection at 192+/- $2 \mathrm{~nm}$. Temperature: $25^{\circ} \mathrm{C}$. Samples: $5 \mathrm{~g} / \mathrm{L}$ DHBC. Assignment of the peaks: PAM- $b$-PAA (A): anisic acid (1), DHBC (2), PAM homopolymer (3); PEO- $b$-PAA (B): system peak (1), DHBC (2), PAA oligomers (3); P(PEGA)-b-PAA (C): MES (1), DHBC (2), PAA oligomers (3); PAM- $b$-PAPTAC (D): Imidazolium (1), DHBC (2), PAPTAC (3). The degree of polymerization of each block is specified on the graph.

The DEM in the series PAM- $b$-PAA (Figure 3A) show three peaks, two sharp at -29 TU (peak 1) and -2 TU (peak 3) and one broad (peak 2) between -7 and -37.5 TU, the latter being assigned to the copolymer of interest. The peak at $-29 \mathrm{TU}$ is assigned to anisic acid (electrophoretic mobility marker) and the one at -2 TU corresponds to homopolymer of PAM. The non-zero electrophoretic mobility of the PAM homopolymer is explained by the incorporation of the negatively charged initiator 4,4'-azobis(4cyanopentanoic) acid. The mass proportion of this PAM population of dead chains has been quantified by external calibration based on time-corrected peak areas using direct injections of PAM solutions of known concentration in the same condition as the DHBC. The proportion of PAM homopolymer 
and $\mathrm{PAM}_{140}-b$-PAA 60 and $8 \%$ for $\mathrm{PAM}_{140}-b-\mathrm{PAA}_{78}$. The copolymer peak is broad and, as expected, the DEM shifts further from zero as the proportion of charged monomer increases in the composition of the copolymers. This can be verified by comparing the average electrophoretic mobility value $\overline{\mu_{e p}^{\text {diblock }}}$ (given in Table 2) which varies between -19.5 TU and -24.1 TU from $\mathrm{PAM}_{140}-b$-PAA $\mathrm{PA}_{40}$.to $\mathrm{PAM}_{140}-b$-PAA $\mathrm{PA}_{78}$ The greater the average molar mass of the polymer, the more dispersed its electrophoretic mobility, as demonstrated by the standard deviation $\sigma_{\mu}$ which varies from $3.9 \mathrm{TU}$ for PAM- $b$-PAA 70-20, to $6.2 \mathrm{TU}$ for PAM- $b$-PAA 140-60.

The DEM of PEO based copolymers are presented in Figure 3B for linear PEO and Figure 3C for PEO grafted polyacrylates (P(PEGA)). Three populations are observed in both series: several small peaks associated with large electrophoretic mobility at $-45 \mathrm{TU}$ are assigned to short oligomers of PAA, the weight percent of which is estimated to be lower than $10 \%$. The sensitivity of the UV detection is too low to conclude about the presence / absence of PEO or P(PEGA) in the DHBC. The least mobile species at -28TU in Figure 3C corresponds to the MES used as mobility marker. The peak at intermediate values of mobility corresponds to the DHBC. The electrophoretic mobility of PEO-b-PAA $\left(\overline{\mu_{e p}^{\text {diblock }}}\right.$ ranging from -28 to -33 TU, Table 2) is significantly closer to zero than that of P(PEGA)- $b$-PAA copolymers ( $\overline{\mu_{e p}^{\text {diblock }}}$ ranging from -36 to-38 TU, Table 2), although the molar masses are close. This is because $\mathrm{P}(\mathrm{PEGA})$, a comb-like polymer, is more compact than linear PEO of the same molar mass. As a consequence, the drag force due to the neutral block is lower for P(PEGA) than for PEO.

As for PAM- $b$-PAPTAC copolymer, PAPTAC homopolymer was detected at about $+43 \mathrm{TU}$, as a shoulder merged in the copolymer distribution, only for the $D P_{\text {neutral }} D P_{0}$ equal to $140 / 100$ and $70 / 57$ samples. Figure 3D displays DEM ranged between +22 and +41 TU, with higher effective mobilities for the DHBC of highest charge. Comparison of PAM- $b$-PAA series with PAM- $b$-PAPTAC series illustrates the importance of the nature of the blocks on the drag effect of the neutral block. This effect is discussed in more detail in section 4.2. 
As a general trend, electrophoretic mobilities of DHBC are always closer to zero than those of the homopolyelectrolyte which are: $\mu_{\mathrm{PAA}}=-42 \mathrm{TU} ; \mu_{\mathrm{PAPTAC}}=+44 \mathrm{TU}$, and the electrophoretic mobility of the DHBC increases as the proportion of charged monomers in the DHBC increases (see Figure SI 20). In terms of EM dispersion, the least dispersed series is the $\mathrm{P}(\mathrm{PEGA})-b-\mathrm{PAA}$, with relative standard deviation of EM $\sigma_{\mu} / \overline{\mu_{e p}^{\text {diblock }}}$ between $6.5 \%$ to $9 \%$, followed by the PEO-b-PAA $\left(\sigma_{\mu} / \overline{\mu_{e p}^{\text {diblock }}} \sim 5 \%\right.$ to $16 \%$ ) and the most disperse series is the PAM- $b$-PAA series with $20 \%$ to $30 \%$ relative standard deviation of the electrophoretic mobility. This dispersion in mobility results from both the level of control of the polymerization, and from the spatial extension of the polymer in the solvent. It can be explained by the chemistry of the RAFT polymerization (chain transfer agent R-SC(S)Z where $\mathrm{Z}$ is the activating group and $\mathrm{R}$ is the leaving group), for which the polymerization of acrylates is better controlled by dithiobenzoates (Z: -SC(S)Ph) (Đ < 1.2) than by ethyl xanthate (Z: -SC(S)OEt) (Đ > 1.3). Besides, the 2-phenylacetate ester of PEO is a better homolytic leaving group than PAM ${ }^{36}$ Furthermore, the synthesis of PAM- $b$-PAA cumulates two successive RAFT/MADIX polymerizations of AM and AA, whereas the synthesis of PEO- $b$-PAA starts from a narrow PEO-CTA $(Đ=1.04)$ (PEO obtained by anionic polymerization) to perform a single RAFT polymerization of AA. This is consistent with the higher dispersity of PAM- $b$-PAA compared to PEO- $b$-PAA ${ }^{31}$. In addition, in reversible-deactivation radical polymerization $^{37}$, at full conversion, the dispersity as defined by the ratio of the weight average molar mass over the number average molar mass, decreases when the $D P$ increases $^{38}$ :

$Ð=1+\frac{1}{D P}+\frac{1}{C_{e x}}$

where $D P$ is the targeted polymerization degree and $C_{\text {ex }}$ is the degenerative chain transfer constant between dormant and active chains ${ }^{39}, 40$, which is consistent with a higher dispersity for a shorter poly(acrylic acid) block in PEO-b-PAA.

To get a better description of the copolymer distribution, it would be interesting to get a distribution of a new parameter which is directly related to the chemical composition of the copolymer instead of the 
374 electrophoretic mobility, which is not linearly dependent on the copolymer composition. This is the 375 purpose of the two following sections.

\begin{tabular}{|c|c|c|c|c|c|c|c|c|}
\hline & & $\begin{array}{c}\text { DP } \text { neutral }^{-} \\
\text {DPo }_{0}\end{array}$ & $\begin{array}{c}\mu_{e p}^{\text {diblock }} \\
\text { peak } \\
\max \\
\text { (TU) }\end{array}$ & $\begin{array}{c}\overline{\mu_{e p}^{\text {diblock }}} \\
\text { integration } \\
\text { (TU) }\end{array}$ & $\begin{array}{c}\sigma_{\mu} \\
(\mathrm{TU})\end{array}$ & $\begin{array}{l}X_{\text {exp }} \\
\text { peak } \\
\max \end{array}$ & $\begin{array}{c}X_{\text {exp }} \text { by } \\
\text { integration }\end{array}$ & $\sigma_{X_{\text {exp }}}$ \\
\hline \multirow{4}{*}{$\frac{\pi}{4}$} & & $70-20$ & -22 & -19.9 & 3.89 & 0.61 & 1.12 & 0.45 \\
\hline & & $140-40$ & -19.1 & -19.5 & 5.44 & 0.74 & 1.26 & 0.73 \\
\hline & & $140-60$ & -23.3 & -21.4 & 6.19 & 0.58 & 1.05 & 1.0 \\
\hline & & $140-78$ & -25.3 & -24.1 & 5.68 & 0.41 & 0.99 & 0.56 \\
\hline \multirow{4}{*}{\multicolumn{2}{|c|}{ 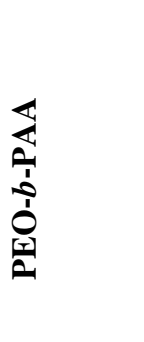 }} & $105-20$ & -31 & -30.2 & 4.83 & 0.28 & 0.33 & 0.12 \\
\hline & & $105-30$ & -33.8 & -33.2 & 2.45 & 0.18 & 0.21 & 0.06 \\
\hline & & $210-40$ & -28 & -28.4 & 1.36 & 0.40 & 0.41 & 0.06 \\
\hline & & $210-50$ & -30.5 & -30 & 1.97 & 0.30 & 0.34 & 0.64 \\
\hline \multirow{2}{*}{ 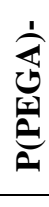 } & & $21-11.5$ & -37.6 & -38.6 & 3.61 & 0.059 & 0.043 & 0.09 \\
\hline & $\frac{1}{b}$ & $44.9-22.2$ & -37 & -36.6 & 2.41 & 0.079 & 0.098 & 0.08 \\
\hline \multirow{4}{*}{\multicolumn{2}{|c|}{ 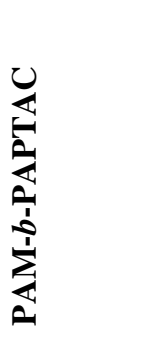 }} & $70-30$ & 34.6 & 33.9 & 3.18 & 0.19 & 0.25 & 0.12 \\
\hline & & $70-60$ & 39.1 & 37.1 & 3.2 & 0.08 & 0.11 & 0.05 \\
\hline & & $140-60$ & 34.2 & 32 & 4.1 & 0.21 & 0.32 & 0.39 \\
\hline & & $140-120$ & 38.5 & 36.3 & 4.1 & 0.09 & 0.164 & 0.13 \\
\hline
\end{tabular}

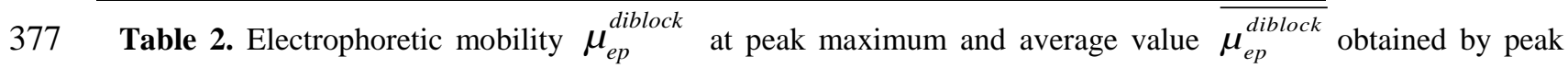

378 integration, standard deviation of the electrophoretic mobility distribution $\sigma_{\mu}, X_{\exp }$ value at peak maximum and

379 average value $\overline{X_{\text {exp }}}$ obtained by peak integration, standard deviation of the $X_{\text {exp }}$ distribution $\sigma_{\text {Xexp }}$ of all DHBC 

$\overline{\mu_{e p}^{\text {diblock }}}$ and $\sigma_{\mu}$ for PAM- $b$-PAA.

\subsection{Change of variable from $\mu_{e p}$ to the retardation parameter $X_{e x p}$}

384 The drag effect of the neutral block can be expressed by the retardation parameter $X_{\text {exp }}$ defined as :

$$
X_{\exp }=\frac{\mu_{e p}^{0}-\mu_{e p}^{\text {diblock }}}{\mu_{e p}^{\text {diblock }}}=\frac{\mu_{e p}^{0}}{\mu_{e p}^{\text {diblock }}}-1
$$
neutral block. It is positive and increases as the drag effect increases.
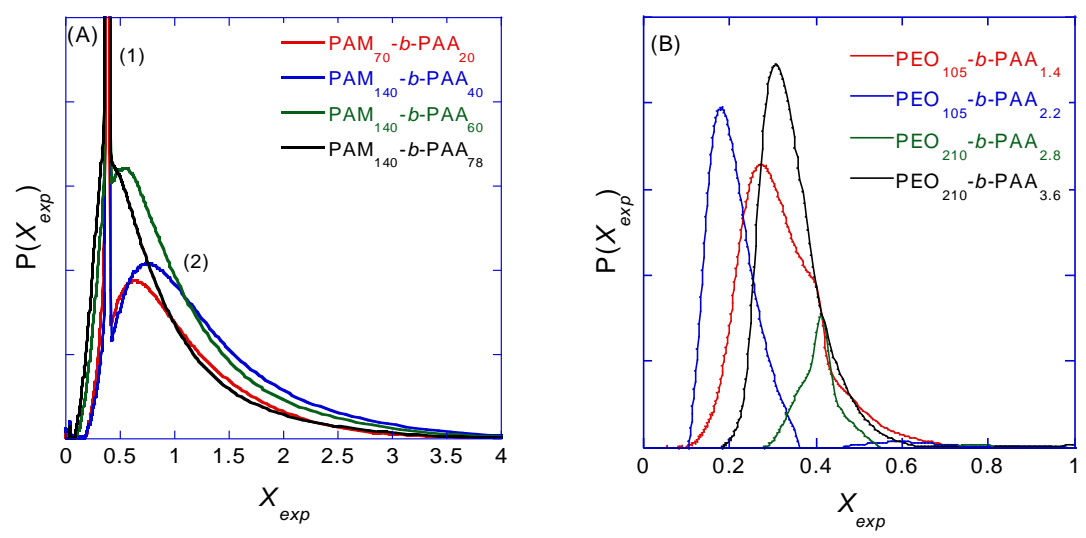
392

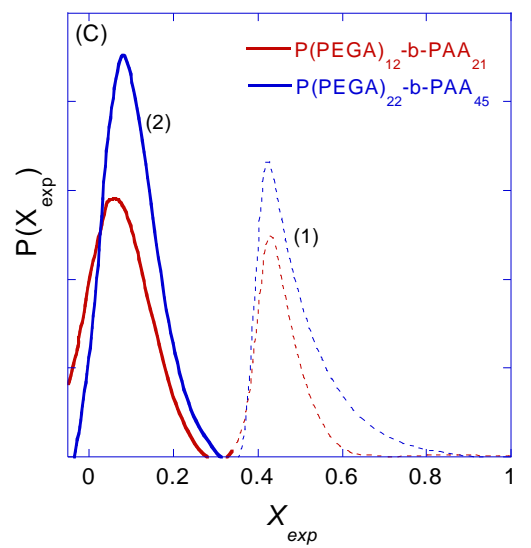

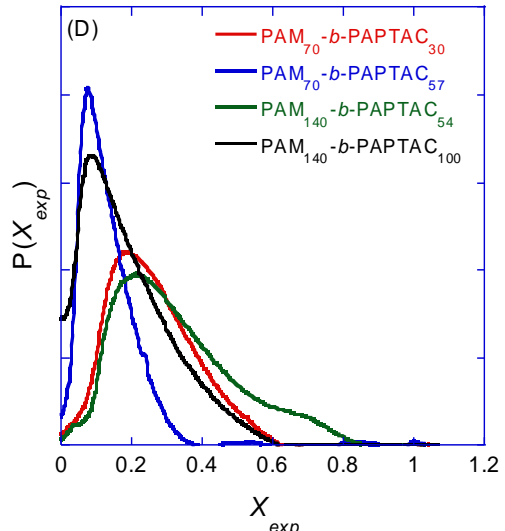

393 Figure 4. Distribution of retardation parameter $X_{\text {exp }}$ for PAM- $b$-PAA (A) PEO- $b$-PAA (B), P(PEGA)$394 b$-PAA (C), and PAM- $b$-PAPTAC (D). Experimental conditions as in Figure $3 X_{\exp }$ was determined using 395 eq. (8), eq. (12) and $\mu_{\mathrm{ep}, \mathrm{PAA}}^{0}=-42 \mathrm{TU} ; \mu_{\mathrm{ep}, \mathrm{PAPTAC}}^{0}=44$ TU. Assignment of the peaks: PAM- $b$-PAA (A): anisic acid (1), DHBC (2); P(PEGA)-b-PAA (C): MES (1), DHBC (2). The degree of polymerization of each block is specified on the graph.

The new experimental variable, $X_{\text {exp }}$, not only points out the friction due to the neutral block but is also more directly related to the composition of the DHBC. Introducing equation (9) in the various expressions of the electrophoretic mobility (equations 1 to 3 ), $X_{\text {exp }}$, can be expressed as a function of the ratio between the degrees of polymerization of the neutral $D P_{\text {neutral }}$ and the charged blocks $D P_{0}$. Taking into account the hydrodynamic coupling (model 1), $X_{\text {exp }}$ reads:

$$
X_{\text {model }, 1}=\frac{1}{N_{b l o b}}=\frac{\alpha}{D P_{0}}
$$

$$
X_{\bmod e l, 1}=\alpha_{1} \frac{D P_{\text {neutral }}}{D P_{0}}
$$


408 with $\alpha_{1}=\frac{b_{1} b_{K_{1}}}{b_{0} b_{K_{0}}}$

409 where $b_{l}$ is the neutral monomer size. The Kuhn statistical segment length (which is twice the persistence

410 length) is a measure of the polymer stiffness. Parameter $\alpha_{l}$ in Equation (10b) is a relative friction

411 coefficient and it is non-dimensional. Since the polyelectrolyte is generally stiffer than the neutral block,

$412 \alpha_{l}$ is often much smaller than unity ${ }^{15}$.

413 As for model 2, it is clear from equation (2) that the $X$ parameter is directly expressed as the ratio of the

414 hydrodynamic radius of each block:

$415 \quad X_{\bmod e l, 2}=\frac{R_{h}^{\text {neutral }}}{R_{h}^{0}}$

416 which can be rewritten as a function of the degrees of polymerization of each block by:

$417 \quad X_{\operatorname{model}, 2}=\frac{C_{1} D P_{\text {neutral }}^{a_{1}}}{C_{0} D P_{0}^{a_{0}}}$

418 where $C_{l}$ (resp. $C_{2}$ ) and $a_{l}$ (resp. $a_{2}$ ) are, respectively, the prefactors and exponents for the neutral (resp.

419 charged) block in the relationship between $R_{h}$ and $D P$. Note that $a_{0}$ and $a_{l}$ are supposed to be close to

$420 \quad 0.5-0.6$ for coil conformations, and slightly higher for more extended conformations.

421 As for model 3 (see equation (3)), the $X$ parameter is directly expressed as:

422

$$
X_{\text {model }, 3}=\frac{2 R_{h}^{\text {neutral }} \ln D P_{0}}{b_{0} D P_{0}}
$$

423 Injecting $R_{h}^{\text {neutral }}$ in equation (9a) leads to:

$$
X_{\bmod e l, 3}=\frac{2 C_{1} D P_{\text {neutral }}^{a_{1}} \ln D P_{0}}{b_{0} D P_{0}}
$$


Finally, equations (10b) (11b) and (12b) demonstrates that, whatever the considered model, the $X$ parameter is related to a compositional ratio between the neutral and the charged blocks with, however, different scaling dependences with the $D P$ of each block, and with an additional logarithmic term in model 3. It is worth noting that in the case of hydrodynamic coupling (model 1), the newly introduced variable varies linearly with the ratio of degree of polymerization of the two blocks.

The distributions of the $X_{\exp }$ parameter are simply deduced from the distribution of electrophoretic mobility using the following equation (Figure 2 , step 4$)^{11}$ :

$P\left(X_{\text {exp }}\right)=\left|\frac{1}{\frac{\partial X_{e p}}{\partial \mu_{e p}}}\right| P\left(\mu_{e p}\right)=\mu_{e p}^{2} P\left(\mu_{e p}\right)$

All the $X_{\text {exp }}$ distributions are presented in Figure 4. Since $\mu_{e p}$ and $X_{\exp }$ are not linearly related, the change of the variable from $\mu_{e p}$ to $X_{\exp }$ modify the form of the distribution. The different moments characterizing the distribution of $X_{\exp }$ are calculated using similar relations as equations (6) and (7) and are reported in Table 2.

$X_{\text {exp }}$ range varies between $0.1-3$ for PAM- $b$-PAA, $0.1-0.7$ for PEO- $b$-PAA, 0-0.3 for P(PEGA)- $b$-PAA and 0-0.8 for PAM- $b$-PAPTAC. The dispersion of the retardation parameter $X_{\exp }$ expressed as $\sigma_{X_{\text {exp }}}$ values (Table 2) follows the following order: P(PEGA)- $b$-PAA $<$ PEO- $b$-PAA $\sim$ PAM- $b$-PAPTAC $<$ PAM- $b$-PAA. The dispersion of the retardation parameter cannot be interpreted as a dispersity in molar mass or in composition since the retardation parameter will change with these chemical features in a way that depends on the conformation of the blocks. So, a further step is needed to get the composition dispersion. 


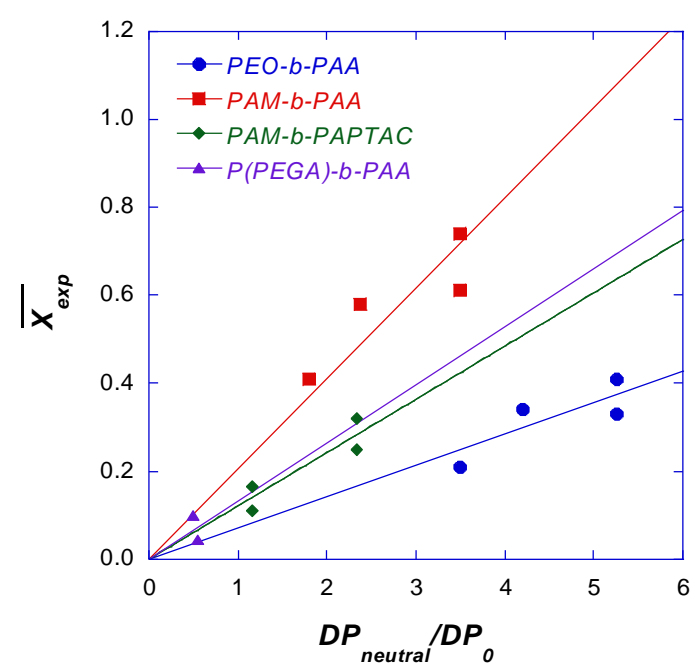

446 Figure 5. Plot of $\overline{X_{\exp }}$ against $\frac{D P_{\text {neural }}}{D P_{0}} \cdot \overline{X_{\exp }}$ was determined by using eq.(6) after replacing $\mu_{e p}$ by $447 X_{\text {exp. }} \frac{D P_{\text {neutral }}}{D P_{0}}$ was obtained by NMR (see Table 1). Least-square linear regressions provide the following experimental slopes: $0.071+/-0.005$ for PEO-b-PAA;0.12+/-0.01 for PAM- $b$-PAPTAC; $0.13+/-0.01$ for P(PEGA)- $b$-PAA; $0.21+/-0.02$ for PAM- $b$-PAA.

To go further in the interpretation and in the process of the experimental data, it is crucial to identify the model which is best adapted to describe the electrophoretic behavior of the DHBC investigated in this work. To assess the validity of model 1 (hydrodynamic coupling between blocks, see section 2.3), $\overline{X_{\text {exp }}}$ was plotted against $\frac{D P_{\text {neutral }}}{D P_{0}}$ in Figure 5 for the four DHBC families.

Table 3. Characteristic parameters of neutral and charged blocks constituting the DHBC studied in this work. $M_{w}$ are expressed in $\mathrm{g} / \mathrm{mol}$. 


\begin{tabular}{|c|c|c|c|c|}
\hline & \\
\hline & $b_{1}(\mathrm{~nm})$ & $b_{K_{1}}(\mathrm{~nm})$ & $R_{h}^{\text {neutral }}(\mathrm{nm})^{\mathrm{a}}$ & 457 \\
\hline PAM & 0.25 & $0.6^{42}$ & $0.01447 \times M_{w}^{0.57} 43$ & 458 \\
\hline \multirow[t]{2}{*}{ PEO } & $0.3^{44}$ & $0.74^{44}$ & $0.02398 \times M_{w}^{0.53} 45$ & 459 \\
\hline & $b_{0}(\mathrm{~nm})$ & $b_{K_{0}}(\mathrm{~nm})$ & $R_{h}^{0}(\mathrm{~nm})$ & 460 \\
\hline PAA & 0.25 & $2.5^{46}$ & $0.007906 \times M_{w}^{0.5852}$ & 461 \\
\hline PAPTAC & & approxima & & \\
\hline
\end{tabular}

464 a From Mark-Houwink parameters using ${ }_{R_{h}}=\left(\frac{3[\eta] M}{10 \pi N_{A}}\right)^{1 / 3}$, where $[\eta]$ is the intrinsic viscosity and $N_{a}$ is the 465 Avogadro number. from ref $^{46}$ (see Table 1 and Figure 13(a) herein).

467 The results are consistent with model 1 which predicts a linear dependence of the retardation parameter $468 \overline{X_{\text {exp }}}$ on the ratio of degree of polymerization $\frac{D P_{\text {neutral }}}{D P_{0}}$. The slopes determined from the graph in Figure 4695 correspond to the parameter $\alpha_{1}$ in equation (10b) which can be calculated from equation (10c). 470 Experimentally, the numerical values of the slopes $\alpha_{l, \exp }$ are in the range of $\sim 0.1-0.2$. Taking the characteristic parameters (Kuhn lengths, monomer dimensions) given in Table 3 leads to $\alpha_{l}=0.24$ ( $v s$ $\alpha_{l, e x p}=0.21+/-0.02$ experimentally obtained) for PAM- $b$-PAA, $\alpha_{l}=0.36$ ( $\left.v s \alpha_{l, e x p}=0.071+/-0.005\right)$ for PEO- $b$-PAA, and $\alpha_{l}=0.24$ ( $\left.v s \alpha_{l, e x p}=0.12+/-0.01\right)$ for PAM- $b$-PAPTAC. Theoretical values of $\alpha_{l}$ are in a reasonably good agreement with the experimental ones, knowing the uncertainty on the persistence length (notably for the polyelectrolyte blocks) and monomer sizes. As for P(PEGA)-b-PAA, we only get an estimation of $\alpha_{1, e x p}=0.13$, since the P(PEGA) Kuhn length is not available in the literature. From

477 Figure 5, we can conclude that the linear correlation between $\overline{X_{\exp }}$ and $\frac{D P_{\text {neutral }}}{D P_{0}}$ is confirmed and that model 1 (with hydrodynamic coupling between the two blocks) can be used to transform the $X_{\exp }$ 
distributions into compositional $\frac{D P_{\text {neutral }}}{D P_{0}}$ distributions. As for models 2 and 3 , they lead to poor correlations between theoretical $X_{\text {model, }}$ versus experimental $\overline{X_{\exp }}$ values (see Figure 6).

481 The knowledge of $\alpha_{l}$ provides the last relation necessary to carry on the general scheme presented in 482 Figure 2 to its end and which leads to the distribution of ratio of chemical composition. In practice, we used $\alpha_{1, \exp }$ obtained in Figure 5 together with equation (10b) to transform the data of Figures 4A to 4D into the distributions presented in Figures 7A to 7D, using the following equation:

$$
P\left(\frac{D P_{\text {neutral }}}{D P_{0}}\right)=\frac{P(X)}{\frac{\partial\left(\frac{D P_{\text {neutral }}}{D P_{0}}\right)}{\partial X}}=\alpha_{1} P(X)
$$

486 Since $X_{\text {exp }}$ and $\frac{D P_{\text {neutral }}}{D P_{0}}$ are linearly correlated, the shapes of both distributions are similar. However, reading $\frac{D P_{\text {neutral }}}{D P_{0}}$ axis, which corresponds to a compositional ratio, is more convenient for the practitioners than keeping the $X_{\text {exp }}$ scale. Moreover, and as previously anticipated, since the $\alpha_{l}$ coefficients are different from one DHBC to another, the distribution in $\frac{D P_{\text {neutral }}}{D P_{0}}$ allows a better comparison between 


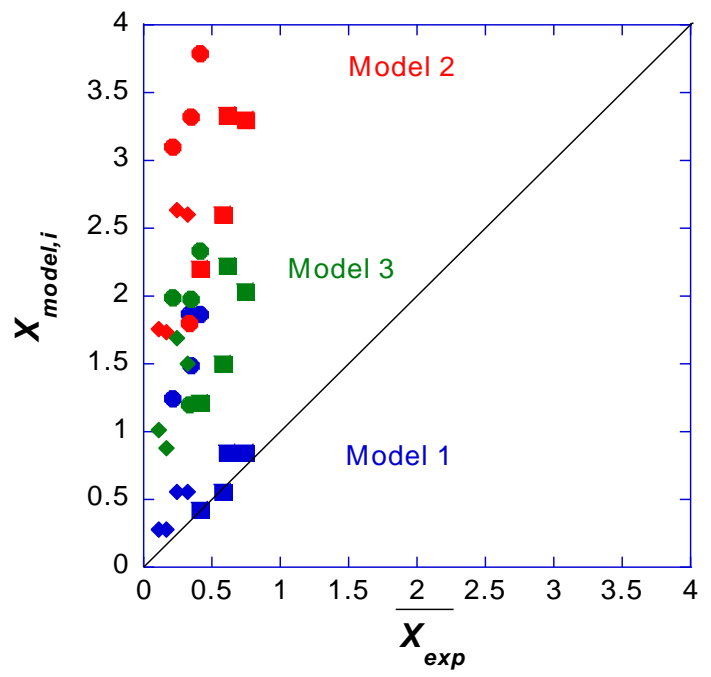

494 Figure 6. Comparison between $X_{\text {model, } \mathrm{i}}$ and $\overline{X_{\mathrm{exp}}}$ for the three different models using theoretical 495 parameters given in Table 3. PAM- $b$-PAPTAC $(\bullet)$; PEO- $b$-PAA $(\bullet)$; PAM- $b$-PAA $(\bullet) . \overline{X_{\text {exp }}}$ was 496 determined by integration of the DHBC peak (in $X$ scale). For all $X_{\text {model, }}$ calculations, theoretical $D P_{\text {neutral }}$ 497 and $D P_{0}$ were used. $X_{\text {mod } e l, 1}$ was determined according to equations (10b) and (10c), $X_{\text {model, }, 2}$ according 498 to equation (11a) and $X_{\text {model, }, 3}$ according to equations (12a), with the characteristic numerical parameters 499 given in Table 3.

500
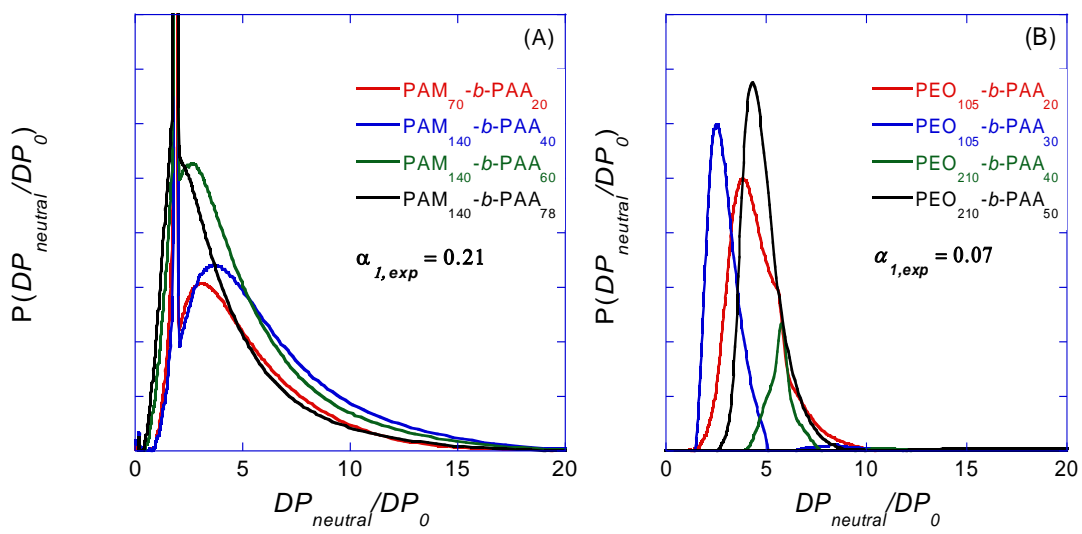

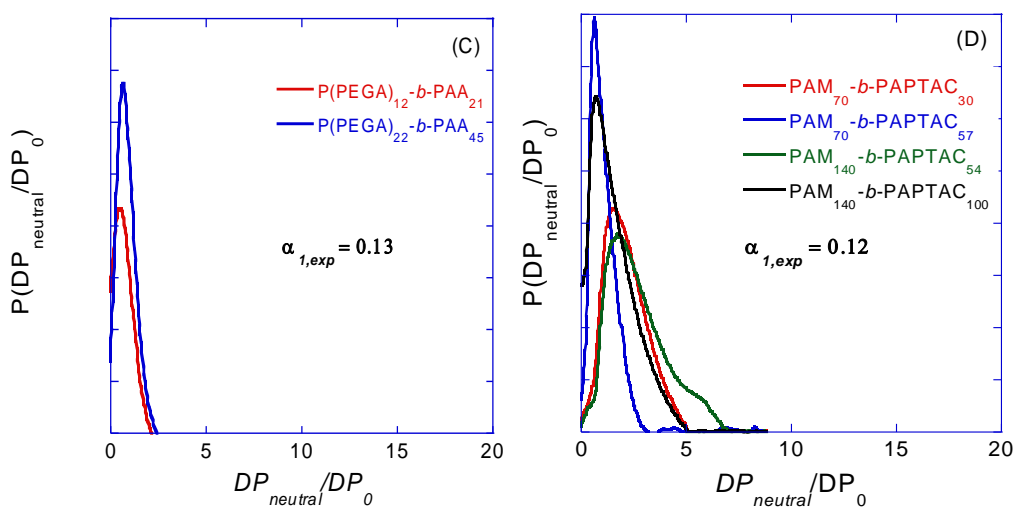

Figure 7. Distribution of composition in terms of the ratio of the degrees of polymerization of both blocks for PAM- $b$-PAA (A), PEO- $b$-PAA (B), P(PEGA)- $b$-PAA (C), and PAM- $b$-PAPTAC (D).

Experimental conditions as in Figure 3. $\frac{D P_{\text {neutral }}}{D P_{0}}$ was determined using eq. (10b) and $P\left(\frac{D P_{\text {neutral }}}{D P_{0}}\right)$ was obtained using eq. (14). In (C), the MES peak has been removed. In (A), the sharp peak is a mobility marker (anisic acid) and was deleted before peak integration.

\section{Conclusion}

In an effort to make information provided by capillary electrophoresis more directly useful for polymer chemists, a protocol was proposed to convert electrophoretic mobility distributions of double hydrophilic block copolymers into distributions of chemical composition ratios. This ratio of composition is expressed as the ratio of the degrees of polymerization of each block $\frac{D P_{\text {neutral }}}{D P_{0}}$. To get this composition ratio, we have introduced the retardation parameter $X$ which takes into account the drag force exerted by the neutral block on the polyelectrolyte. The distribution of $X$ that characterizes a DHBC is readily obtained from the experimental electropherogram and the relation between $X$ and the ratio of $D P$. The latter is available from different models for electrophoretic mobility of composite objects. A linear relation has been found experimentally between the retardation parameter $X$ and the ratio of $D P \mathrm{~s}$, within each of the four families of DHBC studied in this work. This result is consistent with the model of electrophoretic mobility of Long et al. that takes into account hydrodynamic coupling, although the 
prefactors are slightly overestimated. Our experimental findings not only support the theoretical prediction, but also facilitate the last step of data transformation, from distribution of $X$ into distributions of ratios of $D P$.

The dispersions in composition are in the order of: P(PEGA)- $b$-PAA < PEO- $b$-PAA PAM- $b$-PAPTAC $<$ PAM- $b$-PAA. Therefore, we can conclude that in the DHBC families, the PAM block leads to broader composition ratio distributions compared to a PEO block, when associated to a PAA block. Similarly, the P(PEGA) block lead to less disperse composition ratio distributions compared to a PEO block, when associated to a PAA block. Finally, PAA associated with PAM leads to broader composition ratio distributions compared to PAPTAC associated with PAM. The relatively low composition dispersity of the PEO-PAA block copolymer is most likely due to the low dispersity of the PEO block, prepared by anionic polymerization, and the use of a dithiobenzoate chain transfer agent, which has a higher chain transfer constant and thus gives narrower molar mass distributions than the xanthate chain transfer agent used to prepare the PAM-PAA and PAM-PAPTAC block copolymers. The use of a trithiocarbonate chain transfer agent and a relatively short $\mathrm{P}(\mathrm{PEGA})$ block leads to a fairly narrow composition distribution for P(PEGA)-PAA block copolymers.

Finally, the transformation of electrophoretic mobility distributions into composition ratio distributions significantly improved the comparison of the distributions between the different copolymer families, since it takes into account the differences in expansion and drag force according to the chemical nature of the blocks.

\section{Supporting Information.}

541 Synthetic pathway, SEC and NMR characterizations, raw electropherograms are provided for all dibloc 542 copolymers studied in this work. 
544 This work was supported by the MESOPIC project funded by the Agence Nationale de la Recherche 545 (ANR) under grant \# ANR-15-CE07-0005.

\section{References}

547 1. Thevarajah, J. J.; Sutton, A. T.; Maniego, A. R.; Whitty, E. G.; Harrisson, S.; Cottet, H.; 548 Castignolles, P.; Gaborieau, M., Quantifying the Heterogeneity of Chemical Structures in Complex 549 Charged Polymers through the Dispersity of Their Distributions of Electrophoretic Mobilities or of 550 Compositions. Anal. Chem. 2016, 88 (3), 1674-1681.

$551 \quad 2 . \quad$ Morel, A.; Cottet, H.; In, M.; Deroo, S.; Destarac, M., Electrophoretic Behavior of Amphiphilic 552 Diblock Copolymer Micelles. Macromolecules 2005, 38 (15), 6620-6628.

553 3. Sutton, A. T.; Read, E.; Maniego, A. R.; Thevarajah, J. J.; Marty, J. D.; Destarac, M.; Gaborieau, 554 M.; Castignolles, P., Purity of Double Hydrophilic Block Copolymers Revealed by Capillary 555 Electrophoresis in the Critical Conditions. J. Chromatogr. A 2014, 1372C, 187-195.

556 4. Javakhishvili, I.; Jankova, K.; Hvilsted, S., Neutral, Anionic, Cationic, and Zwitterionic Diblock Copolymers Featuring Poly(2-methoxyethyl acrylate)“Hydrophobic” Segments. Polym. Chem.-UK 2013, $4(3), 662-668$.

5. Delplace, V.; Harrisson, S.; Tardy, A.; Gigmes, D.; Guillaneuf, Y.; Nicolas, J., NitroxideMediated Radical Ring-Opening Copolymerization: Chain-End Investigation and Block Copolymer Synthesis. Macromol. Rapid Comm. 2014, 35 (4), 484-491.

6. Nejad, E. H.; Castignolles, P.; Gilbert, R. G.; Guillaneuf, Y., Synthesis of Methacrylate

563 Derivatives Oligomers by Dithiobenzoate-RAFT-Mediated Polymerization. J. Polym. Sci. A1 2008, 46 (6), $564 \quad 2277-2289$.

565 7. Jacquin, M.; Muller, P.; Cottet, H.; Crooks, R.; Théodoly, O., Controlling the Melting of 566 Kinetically Frozen Poly(butyl acrylate-b-acrylic acid) Micelles via Addition of Surfactant. Langmuir 2007, $23(20), 9939-9948$. 

Amphiphilic Diblock Copolymers Synthesized by MADIX Polymerization Process. Macromolecules 2007, 40 (8), 2672-2682.

9. Jacquin, M.; Muller, P.; Cottet, H.; Théodoly, O., Self-Assembly of Charged Amphiphilic Diblock Copolymers with Insoluble Blocks of Decreasing Hydrophobicity: From Kinetically Frozen Colloids to Macrosurfactants. Langmuir 2010, 26 (24), 18681-18693.

10. Anik, N.; Airiau, M.; Labeau, M.-P.; Vuong, C.-T.; Reboul, J.; Lacroix-Desmazes, P.; Gérardin,

C.; Cottet, H., Determination of Polymer Effective Charge by Indirect UV Detection in Capillary Electrophoresis: Toward the Characterization of Macromolecular Architectures. Macromolecules 2009, 42 (7), 2767-2774.

11. Chamieh, J.; Martin, M.; Cottet, H., Quantitative Analysis in Capillary Electrophoresis: Transformation of Raw Electropherograms Into Continuous Distributions. Anal. Chem. 2015, 87 (2), 1050-1057.

12. Long, D.; Ajdari, A., Electrophoretic Mobility of Composite Objects in Free Solution: Application to DNA Separation. Electrophoresis 1996, 17 (6), 1161-1166.

13. Long, D.; Viovy, J.-L.; Ajdari, A., Simultaneous Action of Electric Fields and Nonelectric Forces on a Polyelectrolyte: Motion and Deformation. Phys. Rev. Lett. 1996, 76 (20), 3858-3861.

14. Vreeland, W. N.; Desruisseaux, C.; Karger, A. E.; Drouin, G.; Slater, G. W.; Barron, A. E., Molar Mass Profiling of Synthetic Polymers by Free-Solution Capillary Electrophoresis of DNA- Polymer Conjugates. Anal. Chem. 2001, 73 (8), 1795-1803.

15. Nedelcu, S.; Slater, G. W., Branched Polymeric Labels Used as Drag-Tags in Free-Solution Electrophoresis of ssDNA. Electrophoresis 2005, 26 (21), 4003-4015.

16. Chubynsky, M. V.; Slater, G. W., Theory of End-Labeled Free-Solution Electrophoresis: Is the

591 End Effect Important? Electrophoresis 2014, 35 (5), 596-604.

592 17. Chubynsky, M. V.; Slater, G. W., Electrophoresis of Heteropolymers. Effect of Stiffness. 593 Macromolecules 2015, 48 (16), 5899-5913. 
18. Desruisseaux, C.; Long, D.; Drouin, G.; Slater, G. W., Electrophoresis of Composite Molecular

Objects. 1. Relation between Friction, Charge, and Ionic Strength in Free Solution. Macromolecules 2001, 34 (1), 44-52.

19. Schmidt, B. V. K. J., Double Hydrophilic Block Copolymer Self-Assembly in Aqueous Solution. Macromol. Chem. Phys. 2018, 219 (7), 1700494.

20. Guragain, S.; Bastakoti, B. P.; Malgras, V.; Nakashima, K.; Yamauchi, Y., Multi-StimuliResponsive Polymeric Materials. Chem-Eur. J. 2015, 21 (38), 13164-74.

601 21. Perrier, S., 50th Anniversary Perspective: RAFT Polymerization-A User Guide. Macromolecules 2017, 50 (19), 7433-7447.

22. Matyjaszewski K., X. J., Atom Transfer Radical Polymerization. Chem. Rev. 2001, 100, 29212990.

23. Nicolas, J.; Guillaneuf, Y.; Lefay, C.; Bertin, D.; Gigmes, D.; Charleux, B., Nitroxide-Mediated Polymerization. Prog. Polym. Sci. 2013, 38 (1), 63-235.

24. Destarac, M., Industrial Development of Reversible-Deactivation Radical Polymerization: is the Induction Period Over? Polym. Chem.-UK 2018, 9 (40), 4947-4967.

25. Colfen, H., Double-Hydrophilic Block Copolymers: Synthesis and Application as Novel Surfactants and Crystal Growth Modifiers. Macromol. Rapid Comm. 2001, 22, 219-252.

611 26. Loh, X. J.; del Barrio, J.; Toh, P. P. C.; Lee, T.-C.; Jiao, D.; Rauwald, U.; Appel, E. A.; Scherman, 2012, 13 (1), 84-91.

614 27. Baccile, N.; Reboul, J.; Blanc, B.; Coq, B.; Lacroix-Desmazes, P.; In, M.; Gerardin, C., Ecodesign 615 of Ordered Mesoporous Materials Obtained with Switchable Micellar Assemblies. Angew. Chem. Int. Edit. 2008, 47, 8433-8437.

617 28. Desruisseaux, C.; Drouin, G.; Slater, G. W., Electrophoresis of Composite Molecular Objects. 2. 618 Competition between Sieving and Frictional Effects in Polymer Solutions. Macromolecules 2001, 34 619 (15), 5280-5286. 
29. Long, D.; Dobrynin, A. V.; Rubinstein, M.; Ajdari, A., Electrophoresis of polyampholytes. The J.

Chem. Phys. 1998, 108 (3), 1234-1244.

30. Layrac, G.; Gérardin, C.; Tichit, D.; Harrisson, S.; Destarac, M., Hybrid Polyion Complex Micelles from Poly(Vinylphosphonic Acid)-Based Double Hydrophilic Block Copolymers and Divalent Transition Metal lons. Polymer 2015, 72, 292-300.

31. Taton, D.; Wilczewska, A.-Z.; Destarac, M., Direct Synthesis of Double Hydrophilic Statistical Di- and Triblock Copolymers Comprised of Acrylamide and Acrylic Acid Units via the MADIX Process. Macromol. Rapid Comm. 2001, 22 (18).

32. Bathfield, M.; Warnant, J.; Gérardin, C.; Lacroix-Desmazes, P., Asymmetric Neutral, Cationic and Anionic PEO-Based Double-Hydrophilic Block Copolymers (DHBCs): Synthesis and Reversible Micellization Triggered by Temperature or pH. Polym. Chem.-UK 2015, 6 (8), 1339-1349.

33. Kuhnel, E.; Laffan, D. D.; Lloyd-Jones, G. C.; Martinez Del Campo, T.; Shepperson, I. R.; Slaughter, J. L., Mechanism of Methyl Esterification of Carboxylic Acids by Trimethylsilyldiazomethane. Angew. Chem. Int. Edit 2007, 46 (37), 7075-8.

34. Boursier, T.; Chaduc, I.; Rieger, J.; D'Agosto, F.; Lansalot, M.; Charleux, B., Controlled Radical Polymerization of Styrene in Miniemulsion Mediated by PEO-based Trithiocarbonate Macromolecular RAFT Agents. Polym. Chem.-UK 2011, 2 (2), 355-362.

35. Chaduc, I.; Crepet, A.; Boyron, O.; Charleux, B.; D’Agosto, F.; Lansalot, M., Effect of the pH on the RAFT Polymerization of Acrylic Acid in Water. Application to the Synthesis of Poly(acrylic acid)Stabilized Polystyrene Particles by RAFT Emulsion Polymerization. Macromolecules 2013, 46 (15), 6013-6023.

36. Moad, G.; Rizzardo, E.; Thang, S. H., Living Radical Polymerization by the RAFT Process ? A Third Update. Aust. J. of Chem. 2012, 65 (8), 985.

37. Jenkins, A. D.; Jones, R. G.; Moad, G., Terminology for Reversible-Deactivation Radical Polymerization Previously Called "Controlled" Radical or "Living" Radical Polymerization (IUPAC Recommendations 2010). Pure Appl. Chem. 2009, 82 (2). 
38. Harrisson, S., The Downside of Dispersity: Why the Standard Deviation is a Better Measure of 647 Dispersion in Precision Polymerization. Polym. Chem.-UK 2018, 9 (12), 1366-1370.

648 39. Goto, A.; Fukuda, T., Kinetics of Living Radical Polymerization. Prog. Polym. Sci. 2004, 29 (4), $649 \quad 329-385$.

40. Molina, E.; Warnant, J.; Mathonnat, M.; Bathfield, M.; In, M.; Laurencin, D.; Jerome, C.; Lacroix-Desmazes, P.; Marcotte, N.; Gerardin, C., Drug-Polymer Electrostatic Complexes as New (47), 12839-12844.

41. McCormick, L.; Slater, G.; Karger, A.; Vreeland, W.; Barron, A.; Desruisseaux, C.; Drouin, G., Model. J. Chromatogr. A 2001, 924 (1-2), 43-52.

42. Zhang, W.; Zou, S.; Wang, C.; Zhang, X., Single Polymer Chain Elongation of Poly(Nisopropylacrylamide) and Poly(acrylamide) by Atomic Force Microscopy. J. Phys. Chem. B 2000, 104 (44), 10258-10264.

43. J. Brandrup, E. H. I., E. A. Grulke Polymer Handbook, Fourth Edition, Vol. 2. John Wiley and Sons, Hoboken, New Jersey ed.; 1999; Vol. 2.

662 44. Lee, H.; Venable, R. M.; MacKerell, A. D.; Pastor, R. W., Molecular Dynamics Studies of Polyethylene Oxide and Polyethylene Glycol: Hydrodynamic Radius and Shape Anisotropy. Biophys. J. 2008, 95 (4), 1590-1599.

665

45. Armstrong, J. K.; Wenby, R. B.; Meiselman, H. J.; Fisher, T. C., The Hydrodynamic Radii of Macromolecules and Their Effect on Red Blood Cell Aggregation. Biophys. J. 2004, 87 (6), 4259-4270. 


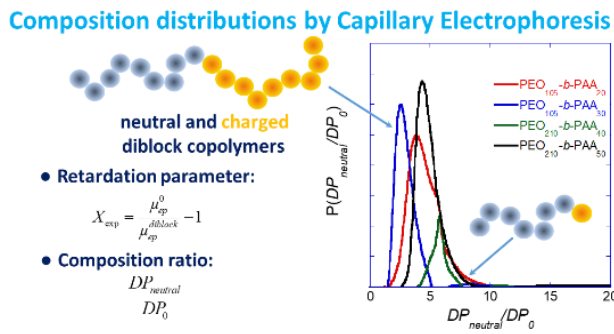

